CASE REPORT

\title{
Alternative methods of lung isolation in cases of pediatric bilateral thoracoscopic surgery
}

\author{
Mark Wigginton ${ }^{1} \&$ Laura Lehrian ${ }^{2}$ \\ 1 Anaesthetic Department, Leeds Children's Hospital, Leeds, UK \\ 2 Division of Pediatric Anesthesia, University of Michigan Health System, Ann Arbor, MI, USA
}

\author{
Keywords \\ pediatrics; anesthesia; CCHS; airway \\ management; one-lung ventilation \\ Correspondence \\ Mark Wigginton, Anaesthetic Department, \\ Leeds General Infirmary, Leeds LS1 3EX, \\ UK \\ Email: dr.mark.wigginton@gmail.com
}

Section Editor: David Polaner

Accepted 5 September 2016

\begin{abstract}
Summary
We compare airway management and lung isolation methods in two pediatric cases of congenital central hypoventilation syndrome undergoing bilateral throacoscopic phrenic-nerve-stimulator surgery. One child received lung isolation using a 7Fr bronchial blocker in conjunction with a 6.0 cuffed endotracheal tube; and the second received a technique of endobronchial intubation using a 3.5 microcuffed tube via the tracheostomy stoma in conjunction with 5.0 cuffed endotracheal intubation; a technique previously undescribed in pediatric patients.
\end{abstract}

doi:10.1111/pan.13012

\section{Introduction}

Congenital central hypoventilation syndrome (CCHS) is a rare condition of abnormal neural crest cell development characterized by alveolar hypoventilation and autonomic dysregulation (1). Many infants with a diagnosis of CCHS have a tracheostomy placed early in life facilitating portable ventilatory support. Around 300 CCHS patients worldwide have been fitted with phrenicnerve-stimulators. This operation is facilitated by lung isolation allowing good visualization of the nerve on the pericardium.

In this report we contrast standard and alternative management methods in two children undergoing bilateral thoracoscopic phrenic-nerve-stimulator placement for CCHS treatment.

\section{Cases}

Both cases underwent gaseous induction via existing tracheostomy followed by conversion to a total intravenous anesthetic technique.

In Case 1, a 9-year-old female, lung isolation was achieved using a 6.0 cuffed oral endotracheal tube (ETT), the cuff distal to the existing tracheostomy stoma; in conjunction with $7 \mathrm{Fr}$ bronchial blocker placed in the right and subsequently left main bronchus.

In Case 2, a 5-year-old female, isolation was achieved by endobronchial intubation via the tracheostomy site using a 3.5 microcuffed ETT into the right main bronchus followed by oral endotracheal intubation with a 5.0 cuffed ETT; cuff level with the existing tracheostomy site. The tip of the oral ETT was visualized during intubation and observed fitting snuggly in the space adjacent to the transtracheal 3.5 endobroncial tube. An occlusive dressing was applied over the stoma site. See Figure 1. Time taken for induction, securing arterial line and lung isolation with bronchoscopic and clinical confirmation were recorded. In Case 1 this was $42 \mathrm{~min}$; in Case 2, $33 \mathrm{~min}$. In both cases, surgery commenced on the right side; left lateral decubitus position. Both techniques achieved excellent lung isolation and optimum surgical conditions.

Midway through both operations, the patients were turned right lateral decubitus and the contralateral lung isolated. For Case 1, this involved bronchoscopic repositioning of the bronchial blocker. For Case 2, the anesthetic circuit was simply switched to the microcuffed ETT. 

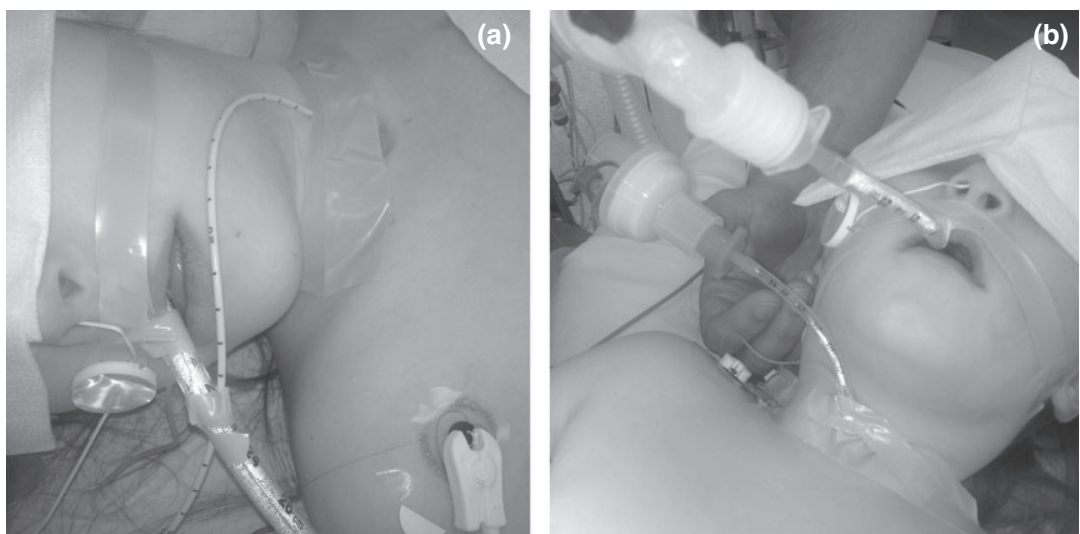

Figure 1 (a) Case 1: oral ETT with cuff and tip distal to trachesotomy stoma with transtracheal 7Fr bronchial blocker. (b) Case 2: right endobronchial transtracheal intubation using a 3.5 microcuffed endotracheal tube with oral endotracheal intubation using a 5.0 cuffed ETT; tip just distal to tracheostomy stoma
Repositioning time in Case 1 was $34 \mathrm{~min}$; in Case 2 this was $8 \mathrm{~min}$. Again, both techniques achieved excellent lung isolation. At completion, the transtracheal devices were removed, patients extubated awake, tracheostomy tubes were reinserted and patients were transferred to the recovery unit. There were no notable postoperative complications.

\section{Discussion}

These cases present the opportunity to directly compare two methods of airway management in patients otherwise treated identically.

Transtracheal endobronchial intubation has previously been described in limited numbers of adult patients. Pediatric double-lumen-tube development is hampered by the difficulty of producing adequate ventilating lumens to fit the narrow trachea. Our technique sought to utilize the extra space afforded by the tracheostomy stoma to provide two useful lumens. Backup equipment for conventional lung isolation using bronchial blocker was immediately available.

Danger of tracheal injury resulting from internal pressure was carefully considered. We used published data to estimate the tracheal and right main bronchus diameter at $8 \mathrm{~mm}$ and $6.7 \mathrm{~mm}$ respectively (2). Use of a cuff pressure monitor along with direct visualization of ETT tip placement adjacent to the transtracheal tube without force or tissue deformation was reassuring. The case 2 method is, however, limited by the size of the main bronchi and trachea compared to the external diameter of the microcuffed tube (2). In the lower trachea, a ventilating lumen equivalent to $3 \mathrm{~mm}$ diameter might be expected but the residual cross-sectional area, accounting for that occupied by the $5 \mathrm{~mm}$ external diameter of the 3.5 endobronchial tube, would be roughly equivalent to a $6.0 \mathrm{~mm}$ ETT. Our patient, aged 5 and $19.1 \mathrm{~kg}$, may represent the lower age/size range for this technique to be safe and successful.
Using a microcuffed ETT to isolate the lung via the tracheostomy stoma reduced anesthetic time by $35 \mathrm{~min}$; the majority saved changing isolation from left to right lung. This method could potentially provide other benefits, allowing variable amounts of positive pressure or even gentle ventilation of the dependent lung by attaching a circuit to the nonventilated ETT. It could facilitate asynchronous lung ventilation if necessary. In the authors' opinion, lung isolation seemed more secure in case 2; bronchial blockers are known to occasionally dislodge or be difficult to position optimally.

\section{Learning points}

- One-lung ventilation can be effectively facilitated by the use of endobronchial intubation via a tracheostomy stoma in conjunction with oral/nasal tracheal intubation down to 5 years of age.

- Careful tube placement with direct vision at the tracheostomy and cuff pressure monitor is advocated to reduce the risk of tracheal injury.

- This technique has theoretical benefits compared with bronchial blockers including the ability to apply dependent lung CPAP or asynchronous ventilation.

\section{Ethics}

No approval required.

\section{Funding}

The study was funded by departmental resources.

\section{Disclosures}

No conflicts of interest. 


\section{References}

1 Rand C, Carroll M, Weese-Mayer D. Congenital central hypoventilation syndrome: a neurocristopathy with disordered respiratory control and autonomic regulation. Clin Chest Med 2014; 35: $535-545$.
2 Hammer G, Fitzmaurice B, Brodsky J. Methods for single-lung ventilation in pediatric patients. Anesth Analg 1999; 89: 1426-1429. 